\title{
Massive MIMO for Tactical Ad-hoc Networks in RF Contested Environments
}

\author{
Asanka Kekirigoda*, Kin-Ping Hui*, Qingqing Cheng ${ }^{\dagger}$, Zhipeng Lin ${ }^{\dagger}$, \\ J. Andrew Zhang ${ }^{\dagger}$, Diep N. Nguyen ${ }^{\dagger}$, Xiaojing Huang ${ }^{\dagger}$ \\ *Defence Science and Technology (DST), Edinburgh, Australia \\ ${ }^{\dagger}$ Global Big Data Technologies Centre, University of Technology Sydney, Sydney, Australia \\ E-mails: \{asanka.kekirigoda; kin-ping.hui\}@dst.defence.gov.au;\{Qingqing.Cheng; \\ Zhipeng.Lin\}@student.uts.edu.au; \{Andrew.Zhang; Diep.Nguyen; Xiaojing.Huang\}@uts.edu.au
}

\begin{abstract}
Survivability of wireless communications segments in tactical military networks is an enormous challenge in the present and future defence forces, especially as these networks usually operate in radio frequency (RF) contested environments. Therefore, it is necessary to develop techniques to provide effective and efficient communication in RF contested environments. Massive multiple-input-multiple-output (MIMO) techniques use a large number of antennas enabling higher degrees of freedom that can improve communications network's survivability and efficiency compared to conventional MIMO or single antenna systems. This paper presents a novel massive MIMO communications system which enhances the throughput of the network, reduces the bit-error-rate and mitigates the interference from high powered jammers. Simulation results in contested environments verify the effectiveness of this system.

Index Terms-Multiple-input-multiple-output (MIMO), Multi-user massive MIMO, Tactical communications, Contested networks, Space-division multiple access (SDMA)
\end{abstract}

\section{INTRODUCTION}

As defence forces worldwide transform into more networkcentric systems in operations, the survivability of tactical network segments becomes critically important to the successful outcome of the mission, especially in hostile environments. In particular, it is the wireless segments that are the most susceptible to radio frequency (RF) attacks. Hence, new techniques are required to maintain the tactical wireless communications links in such challenging conditions.

To overcome these challenges, multiple-input-multipleoutput (MIMO) antenna techniques have been proposed as an effective and efficient alternative to single antenna techniques for tactical wireless communications systems [1]-[5]. The use of spatially separated multiple antennas enables the system to exploit the degrees of freedom to separate not only communication channels but also jammers' channels. Furthermore, MIMO techniques were shown to lower the bit-error-rate (BER) of the tactical links in RF contested environments compared to single antenna systems [6].

Massive MIMO technology can scale up conventional MIMO systems by many orders of magnitude. It has been accepted as one of the most beneficial techniques for the next generation of commercial wireless communications networks, mainly due to its enormous potential in improving the spectral efficiency and energy efficiency [7], [8]. This technique uses multi-user MIMO or space-division multiple access (SDMA) to achieve a multiplexing gain by serving multiple nodes on the same time-frequency resources. In such multi-user massive MIMO systems, the number of antennas in a base station is significantly larger than the number of user equipments or the number of independent spatial streams. This allows for focusing the RF energy into smaller areas of space to produce higher capacity and overall energy efficiency without increasing the transmit power. For military applications, the excess degrees of freedom can also be used to mitigate signals from jammers.

Although massive multi-user MIMO has been widely studied for commercial cellular communications networks, research on its application in military tactical ad-hoc networks is still very limited. We extend multi-user massive MIMO to the network (point-to-multipoint) communications to make it more suitable for wireless tactical ad-hoc networks. In addition to increasing network capacity and improving immunity to jamming, it has the potential to decrease the RF footprint, thereby improving low probability of interception (LPI) and low probability of detection (LPD).

This paper aims to investigate the performance of multiuser massive MIMO for wireless tactical ad-hoc networks in an RF contested environment. We consider a stationary tactical scenario consisting of multiple military vehicles (nodes) and each node is equipped with a massive MIMO antenna system. We also consider multiple stationary jammers sending continuous and random jamming signals in different locations. Novel algorithms are employed both at the transmitter and receiver nodes to exploit the potential of massive MIMO systems for suppressing the RF interference from the jammers, while simultaneously realising SDMA. We then compare the proposed system with other conventional systems. The proposed scheme is shown to be very efficient in suppressing jamming signals, while supporting SDMA communications.

The remainder of the paper is organised as follows. Section II describes the multi-user massive MIMO channel model used in this paper and Section III presents the proposed tactical multi-user massive MIMO system. Section IV describes 


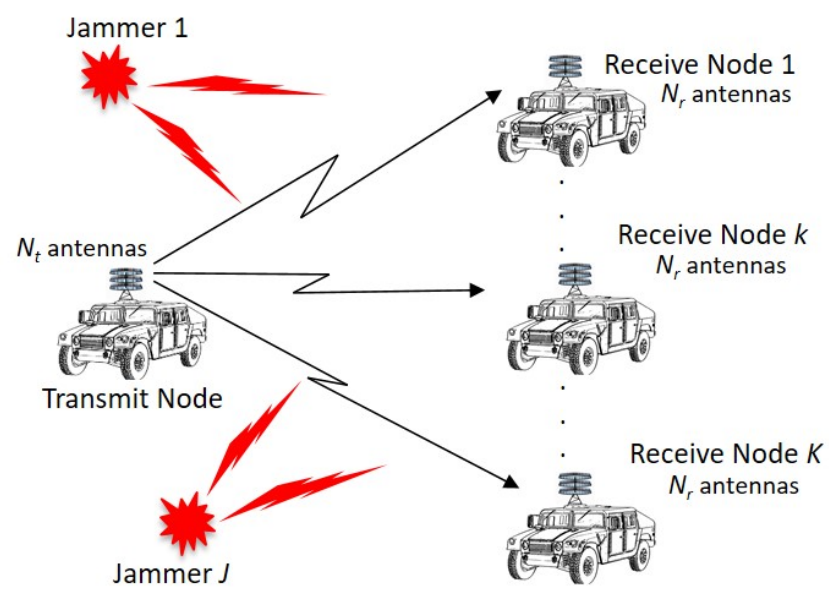

Fig. 1: Multi-user MIMO model for ad-hoc networks.

the tactical scenarios used to verify our system. Section V compares the performance of the proposed system with other systems through numerical simulations, and conclusions are provided in Section VI.

Notation: $(.)^{T},(.)^{*},(.)^{H}$, and $(.)^{-1}$ denote the matrix/vector transpose, conjugate, conjugate (Hermitian) transpose, and inverse operation, respectively.

\section{SySTEM MODEL}

We consider a military ad-hoc network where one node uses SDMA to communicate to multiple other nodes in the presence of high-power jammers. There are $N_{t}$ antennas at the transmit node and $N_{r}$ antennas at the receive node $k$, as illustrated in Fig. 1. We will consider homogeneous nodes in the ad-hoc network, therefore $N_{t}=N_{r}=N$. There are $K$ receive nodes and also $J$ jammers that continuously transmit random jamming signals. The number of spatial data streams for receive node $k$ is $S_{k}$, where $S_{k} \leq N$. The total number of spatial streams is $S=\sum_{k=1}^{K} S_{k}$.

Let us consider a vector signal $\mathbf{x}_{k}$ of length $S_{k}$ being transmitted to receiving node $k$. Let $\mathbf{P}_{k}$ be the precoding matrix applied at transmitter for receiving node $k$, with size of $N \times S_{k}$. The received signal vector of length $N$ at the receive node $k$ is given by

$$
\mathbf{y}_{k}=\mathbf{H}_{k} \mathbf{P}_{k} \mathbf{x}_{k}+\mathbf{H}_{k} \sum_{i \neq k}^{K} \mathbf{P}_{i} \mathbf{x}_{i}+\sum_{j=1}^{J} \mathbf{Z}_{k, j} \mathbf{f}_{j}+\mathbf{n},
$$

where $\mathbf{H}_{k} \in \mathbb{C}^{N \times N}$ denotes the channel matrix between the transmit node and receive node $k ; \mathbf{Z}_{k, j} \in \mathbb{C}^{N_{k} \times 1}$ is the channel between jammer $j$ and receive node $k$; $\mathbf{x}$ and $\mathbf{f}$ stand for the signals transmitted from the transmit node and jammers, respectively; and $\mathbf{n}$ is the background noise which is modelled as an additive white Gaussian noise (AWGN) with independent and identically distributed (i.i.d.) entries of zero mean and variance $\sigma^{2}$. We assume that $\mathbf{H}_{k}$ and $\mathbf{Z}_{k}$ are perfectly known at receive node $k$.
For a transmitter-receiver pair, $\mathbf{H}_{k}$ is given by

$$
\mathbf{H}_{k}=\sum_{l=1}^{L} v_{k, l} \mathbf{a}_{k}\left(\theta_{a, l}\right) \mathbf{a}_{k}\left(\theta_{d, l}\right)^{T},
$$

where $v_{k, l}$ is receive node $k$ 's amplitude of complex value accounting for both signal attenuation and initial phase difference; $\mathbf{a}_{k}\left(\theta_{a, l}\right)$ and $\mathbf{a}_{k}\left(\theta_{d, l}\right)$ are the steering vectors of the transmitter and receiving arrays, respectively. Here $L$ is the total number of multipath between a transmitterreceiver pair (including jammers). We consider a uniform circular array (UCA) in this paper, and $\mathbf{a}_{k}\left(\theta_{a, l}\right)=$ $\left[e^{j \pi \cos \left(\theta_{a, l}\right)}, \ldots, e^{j \pi \cos \left(\theta_{a, l}-2 \pi(N-1) / N\right)}\right]^{T}$, with $\theta_{a, l}$ denoting the angle-of-arrival (AoA) from path $l ; \mathbf{a}_{k}\left(\theta_{d, l}\right)=$ $\left[e^{j \pi \cos \left(\theta_{d, l}\right)}, \ldots, e^{j \pi \cos \left(\theta_{d, l}-2 \pi(N-1) / N\right)}\right]^{T}$, with $\theta_{d, l}$ denoting the angle-of-departure (AoD) from path $l$.

Similarly, for the jammer channel $\mathbf{Z}_{k, j}$, it can be expressed as

$$
\mathbf{Z}_{k, j}=\sum_{l=1}^{L} v_{j, l} \mathbf{a}_{j}\left(\phi_{a, l}\right) e^{j \pi \cos \left(\phi_{d, l}\right)}
$$

where $v_{j, l}$ stands for jammer $j$ 's amplitude of complex value accounting for both signal attenuation and initial phase difference; $\mathbf{a}_{j}\left(\phi_{a, l}\right)=\left[e^{j \pi \cos \left(\phi_{a, l}\right)}, \ldots, e^{j \pi \cos \left(\phi_{a, l}-2 \pi(N-1) / N\right)}\right]^{T}$, with $\phi_{a, l}$ denoting AoA value from path $l$; and $\phi_{d, l}$ denoting AoD from path $l$.

\section{Proposed Tactical Multi-user Massive Mimo SYSTEM}

In this section, we provide an overview of the proposed system. For the receiving node $k$, the received signal after processing can be represented as

$$
\mathbf{q}_{k}=\mathbf{W}_{b k}^{H} \mathbf{W}_{a k}^{H} \times \mathbf{y}_{k},
$$

where $\mathbf{W}_{a k} \in \mathbb{C}^{N_{k} \times S_{k}}$ is mainly for jamming suppression, and $\mathbf{W}_{b k} \in \mathbb{C}^{S_{k} \times S_{k}}$ is for equalization of equivalent channels $\mathbf{W}_{a k}^{H} \mathbf{H}_{k} \mathbf{P}_{k}$.

\section{A. Jamming Mitigation}

The priority of the proposed system is the mitigation of jamming signals. Since jamming signals have high power, it can be more detrimental to the receiver compared to multi-user interference. Hence the first step is to nullify the jamming signals by exploiting the $N$ degrees-of-freedom offered by multiple antennas, so that the received useful signals become orthogonal to the jamming signals after the first-step processing.

Let $\mathbf{Z}_{k}=\left[\mathbf{Z}_{k, 1}, \ldots, \mathbf{Z}_{k, J}\right]^{T}$. To achieve this goal, we compute the null space of $\mathbf{Z}_{k}$ and let $\mathbf{W}_{a k}^{H}$ be a matrix spanning on the null space. The null space can be obtained by computing the singular value decomposition (SVD) of the jamming channel matrix $\mathbf{Z}_{k}$.

Let the left singular vector matrix of $\mathbf{Z}_{k}$ be $\mathbf{U}_{\mathbf{z}}$. The null space $\mathbf{Q}_{k} \in \mathbb{C}^{N \times(N-J)}$ is then obtained from the $(N-J)$ columns of $\mathbf{U}_{\mathbf{z}}$ corresponding to the $(N-J)$ least singular values (having zero values in the ideal case). Since 
$\mathbf{W}_{a k}$ is from the null space of $\mathbf{Z}_{k}$, we have $\mathbf{W}_{a k}^{H} \mathbf{Z}_{k}=0$. Consequently, the jamming signals, $\mathbf{W}_{a k}^{H} \sum_{j=1}^{J} \mathbf{Z}_{k, j} \mathbf{f}_{j}$, are filtered out.

\section{B. Design of $\mathbf{W}_{a k}$, Precoding Matrix $\mathbf{P}$ and $\mathbf{W}_{b k}$}

We adopt eigen-beamforming to design $\mathbf{W}_{\mathbf{a k}}$, which can be represented as

$$
\mathbf{W}_{\mathrm{ak}}=\mathbf{Q}_{\mathrm{k}} \mathbf{B}_{\mathrm{k}}
$$

where $\mathbf{B}_{k}$ is a $(N-J) \times S_{k}$ matrix.

Here multi-user interference is not considered when deciding $\mathbf{B}_{k}$ at receive node $k$. In this case, $\mathbf{B}_{k}$ can be determined by selecting $S_{k}$ eigenvectors corresponding to $S_{k}$ maximal eigenvalues in the SVD of $\left(\mathbf{Q}_{k}^{H} \mathbf{H}_{k} \mathbf{H}_{k}^{H} \mathbf{Q}_{k}\right)$. This method only requires the receive node $k$ to know its own channel, and then feed back $\widetilde{\mathbf{H}}_{k}=\mathbf{W}_{a k}^{H} \mathbf{H}_{k}$ to the transmit node. We denote this method as "Rx Eigen-BF".

For the precoder, we use zero-forcing (ZF) design, which can easily be obtained by channel inversion as

$$
\mathbf{P}^{\mathrm{ZF}}=\left[\mathbf{P}_{\mathbf{1}}, \cdots, \mathbf{P}_{\mathbf{K}}\right]=\widetilde{\mathbf{H}}^{-\mathbf{1}} \boldsymbol{\Lambda},
$$

where $\boldsymbol{\Lambda}$ is a diagonal matrix accounting for power normalization and power loading (if desired). We apply $\boldsymbol{\Lambda}$ to normalize the power of each column in $\mathbf{P}^{\mathrm{ZF}}$ to be $P_{T} / S$ here, where $P_{T}$ is the total transmission power.

When the precoder $\mathbf{P}$ is determined, the combiner $\mathbf{W}_{\mathbf{b k}}$ for UE $k$ can be designed based on the combined channel given by

$$
\breve{\mathbf{H}}_{k}=\mathbf{W}_{\mathbf{a k}} \mathbf{H}_{\mathbf{k}} \mathbf{P}_{\mathbf{k}} \text {. }
$$

The simplest ZF equalizer is given by $\mathbf{W}_{\mathbf{b f}}=\left(\breve{\mathbf{H}}_{\mathbf{k}}\right)^{-\mathbf{1}}$. Note that the equivalent channel matrix $\breve{\mathbf{H}}_{k}$ will be diagonal when there is no channel estimation error.

\section{TACTICAL SCENARIO}

We consider a scenario representing a stationary armoured troop in a tactical ad-hoc communication network in an open terrain and RF contested environment as represented in Fig. 2. The scenario consists of 5 stationary ground vehicles (nodes) with multiple antennas on each of them. Each node transmits to every other node in the network using SDMA and time-division-multiple-access (TDMA). The channel in each communication link is modelled as slow (quasi-static) Rician fading channel. We consider two stationary jammers $(J=2)$ as well. The notional positions of the five nodes and the jammers are shown in Fig. 2.

We use the following parameters to simulate the scenario:

- Ratio of jamming transmit power to signal of interest power $=1$;

- Use 16 quadrature amplitude modulation (16 QAM) with no FEC coding;

- Narrowband system with single carrier modulation;

- Nodes are equipped with 8 antennas $(N=8)$, unless otherwise stated;

- All antennas are isotropic and arranged as a uniform circular array;

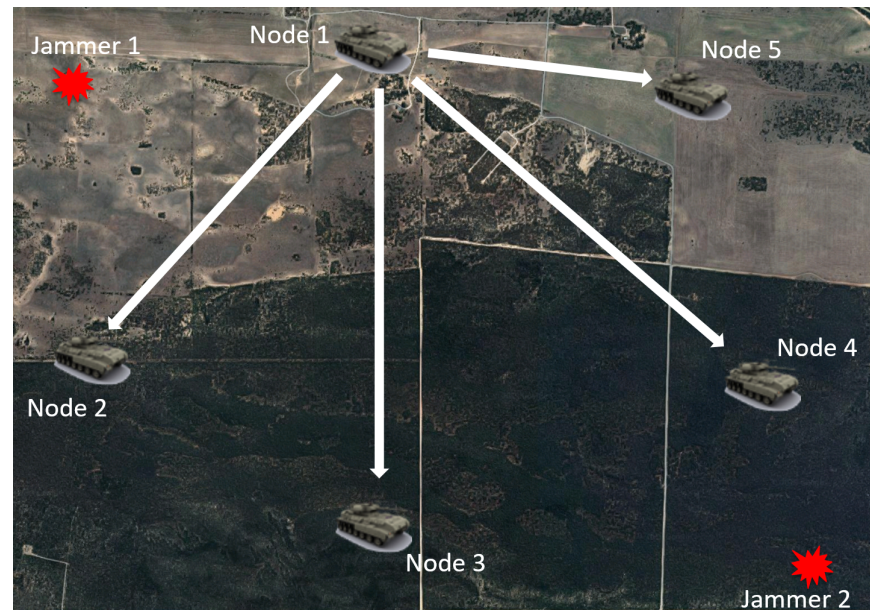

Fig. 2: Node and jammer locations in the tactical scenario.

- Transmit node sends 1 spatial stream to each of 4 receivers,

- The total number of multipath between a pair of transmit node (including jammers) and receive node $=5$;

- Rician factor, which is the power ratio between the lineof-sight (LoS) and non-LoS (NLoS) path is 5 and 10 for receivers and jammers, respectively.

The AoAs of multipath for the receiver and jammer are uniformly distributed in the range of $\left[\theta_{a, k}-\alpha_{a}, \theta_{a, k}+\alpha_{a}\right]$ and $\left[\phi_{a, j}-\alpha_{a}, \phi_{a, j}+\alpha_{a}\right]$, where $\theta_{a, k}=120$ and $\phi_{a, j}=$ 150 degrees are the AoAs of the LoS path for receiver $k$ and jammer $j$, respectively, and the angle spread $\alpha_{a}$ is 80 degrees. The AoDs of multipath for the receiver and jammer are uniformly distributed in the range of $\left[\theta_{d, k}-\alpha_{d}, \theta_{d, k}+\right.$ $\left.\alpha_{d}\right]$ and $\left[\phi_{d, j}-\alpha_{d}, \phi_{d, j}+\alpha_{d}\right]$, where $\theta_{d, k}=\phi_{d, j}=80$ degrees are AoDs of the LoS path for receiver $k$ and jammer $j$, respectively, and the angle spread $\alpha_{d}$ is 360 degrees.

\section{Simulation Results and Discussion}

This section presents the simulation results to validate the performance of our proposed scheme, using the metrics of bit error rate (BER) and sum-rate upper bound. The sumrate upper bound provides an upper bound for the multiuser capacity for the given equivalent channel $\widetilde{\mathbf{H}}_{k}$, without considering specific design of $\mathbf{P}$ and $\mathbf{W}_{\mathbf{b k}}$. It is given by

$$
R=\log _{2}\left|\mathbf{I}+\frac{P_{T}}{N_{T} \sigma^{2}} \sum_{k=1}^{K} \mathbf{W}_{a k} \mathbf{H}_{\mathbf{k}}\left(\mathbf{W}_{\mathbf{a k}} \mathbf{H}_{\mathbf{k}}\right)^{\mathbf{H}}\right| .
$$

Since we have not been able to find existing work on the same topic with our paper, we provide comparison with a benchmark algorithm based on the sum-rate maximisation by referring to the successive interference cancellation (SIC) method in Section IV-B in [9]. It is combined with the ZF precoder proposed in this paper and we compare both cases when the jamming is present and absent. We denote the two cases as "SIC, No Anti-Jam" (jamming mitigation technique is not used) and "SIC, No Jam", respectively. Our proposed 

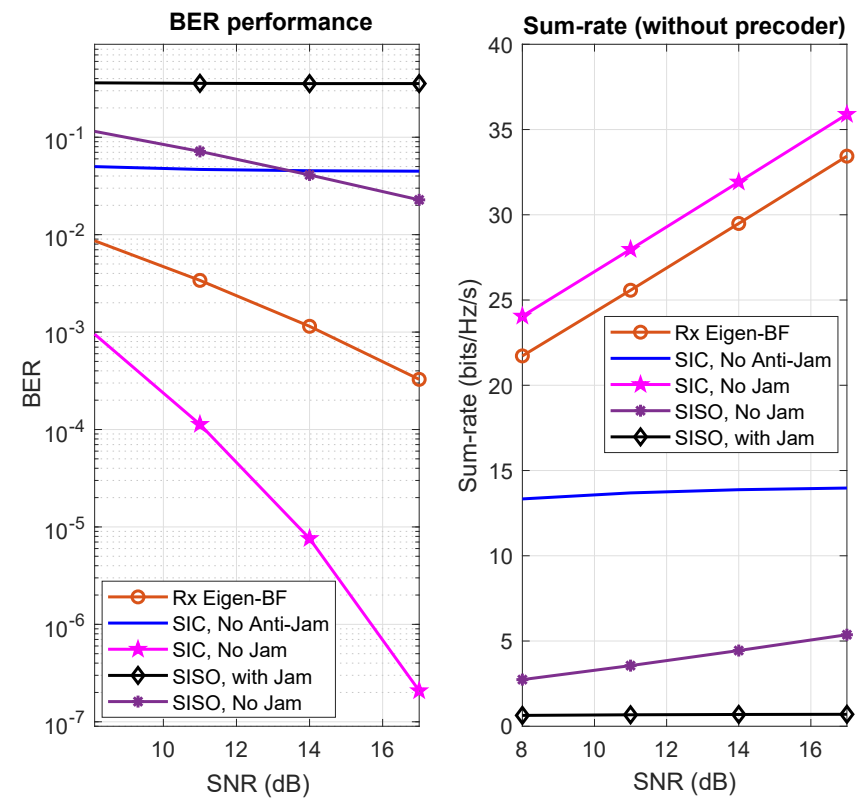

Fig. 3: BER and sum-rate versus SNR for 8 antennas.

system is denoted as "Rx Eigen-BF" and is always simulated in the presence of jamming.

Fig. 3 depicts the variations of BER and sum-rate performance with signal-to-noise ratio (SNR) for an 8 antenna system. The BER and sum-rate for a single-input singleoutput (SISO) system both in the presence and absence of jamming are also simulated for comparison. These two are denoted as "SISO, with Jam" and "SISO, No Jam", respectively. We can observe that the BER is extremely high and the sum-rate is low for SISO and SIC systems with
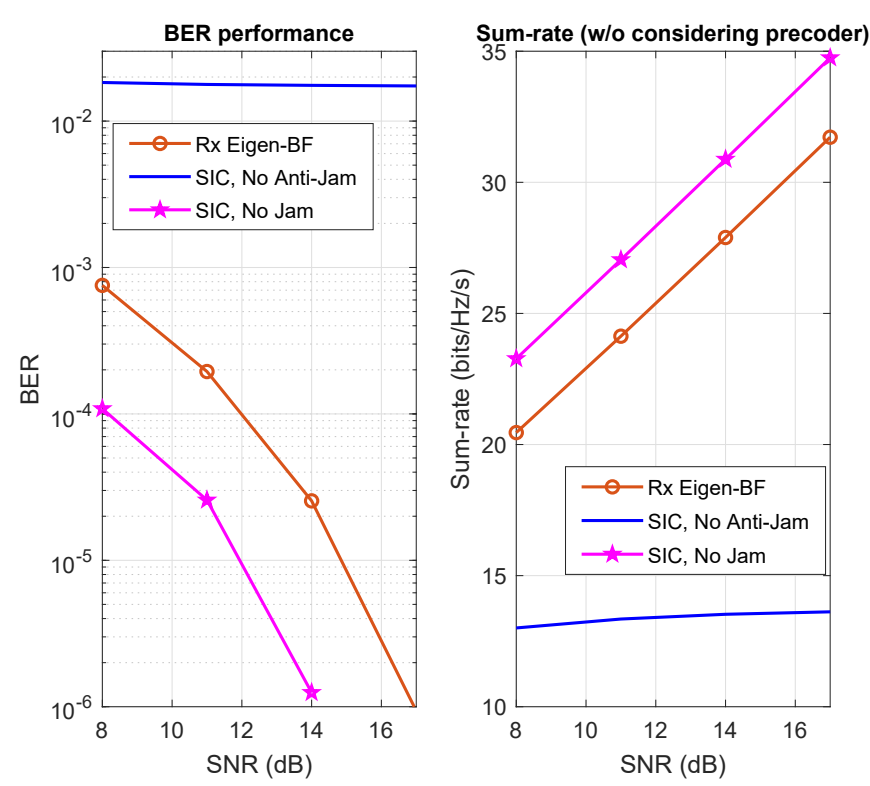

Fig. 4: BER and sum-rate versus SNR for 16 antennas.
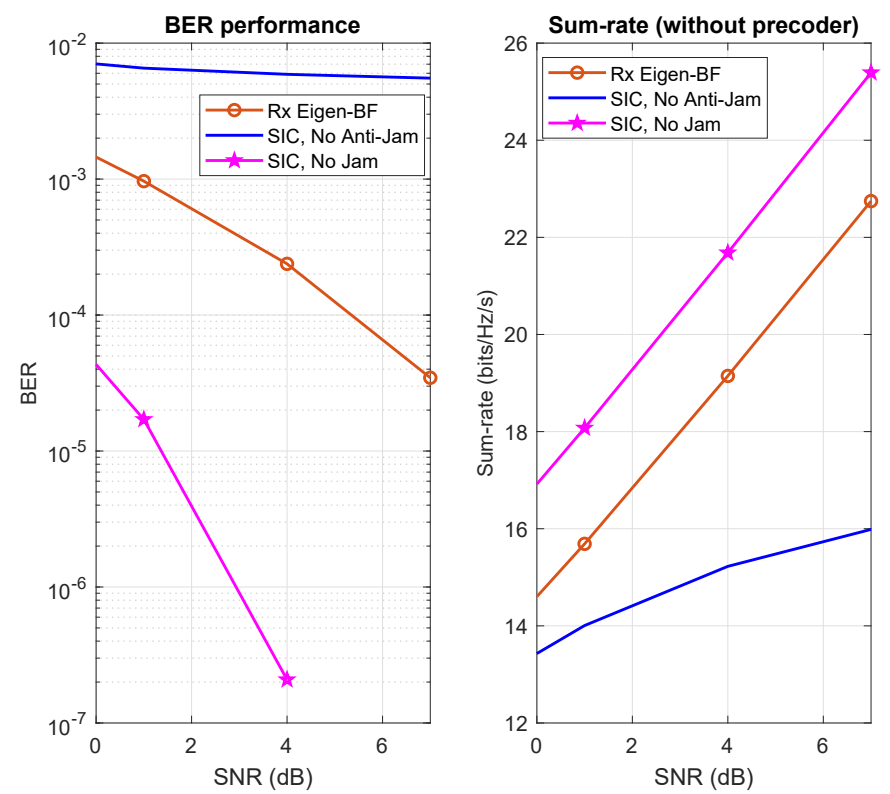

Fig. 5: BER and sum-rate versus SNR for 32 antennas.

jamming. Hence, these systems cannot be used to achieve effective communication. Even in the absence of jamming, the SISO system is still inferior to our proposed system. The main reason is that in MIMO systems multiple antennas at the receiver and transmitter provide diversity gain to each receiver compared to the SISO system, where there is no diversity gain.

Figs. 4 to 6 depict the variations of BER and sum-rate performance with SNR for different numbers of antennas from 16 to 64 . From these figures as well as from Fig. 3, we observe the following:
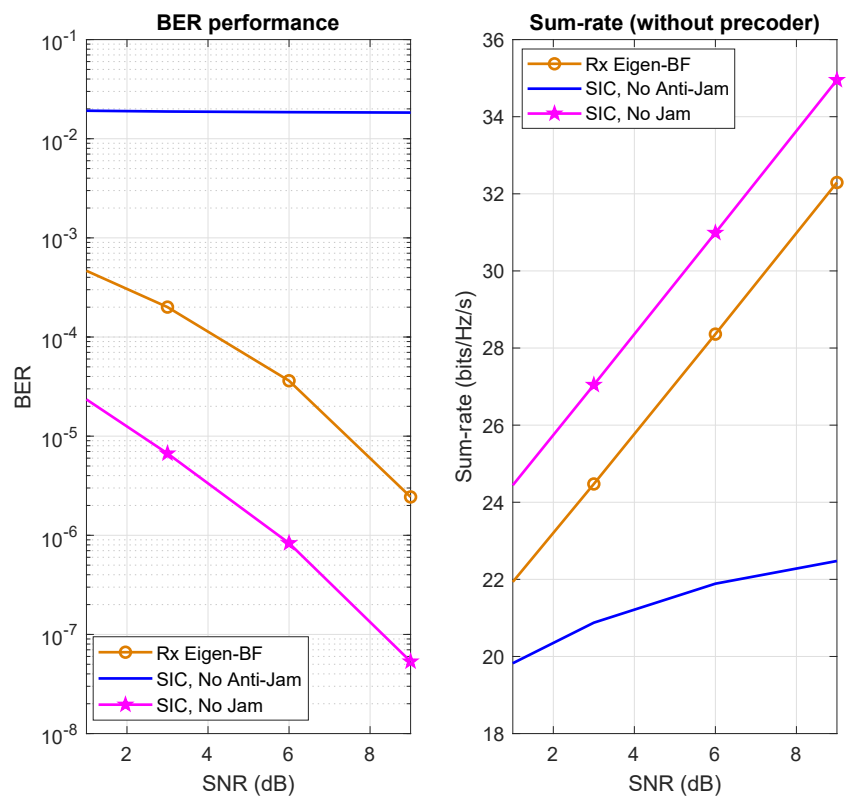

Fig. 6: BER and sum-rate versus SNR for 64 antennas. 


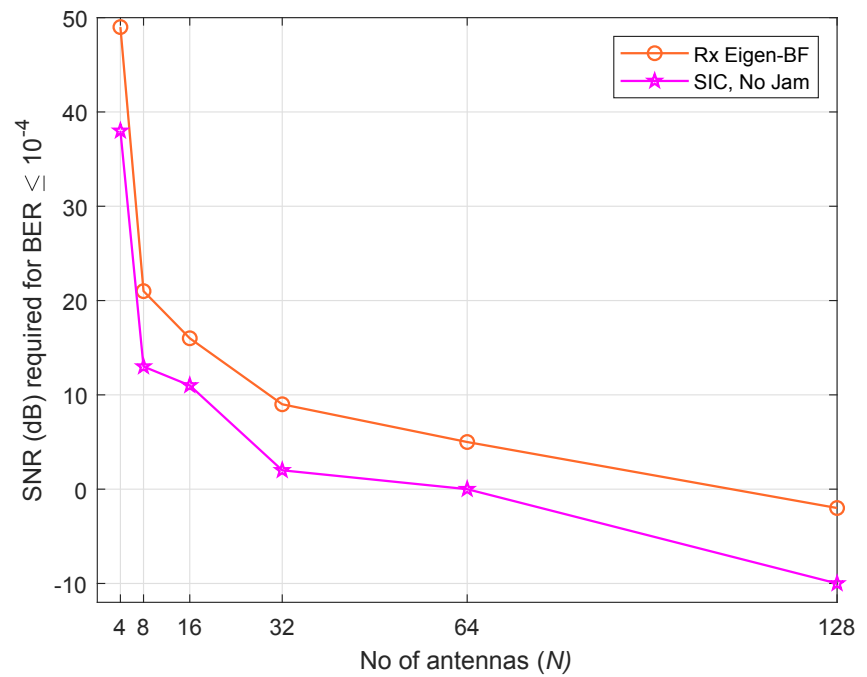

Fig. 7: SNR required for a BER $\leq 10^{-4}$ for different number of antennas $(N)$.

- In the presence of jamming signal, conventional receiver such as "SIC, No Anti-Jam" fails to work. Our proposed jamming mitigation algorithm can effectively suppress jamming, while still supporting multi-user MIMO.

- Jamming causes performance loss, even with jamming mitigation technique employed, compared to the case without having jamming signals. This can be seen by the better performance of SIC with no jamming compared to our proposed system in the presence of jamming.

- Significant capacity gain achieved by MIMO as can be seen from the sum-rates plots. The sum-rate also increases with the number of antennas, at the ratio of approximately $3 \mathrm{~dB}$ per 2 to the power of the number of antennas for "SIC, No Jam" and "Rx Eigen-BF".

Fig. 7 depicts the SNR required for a BER of $\leq 10^{-4}$ for different numbers of antennas from 4 to 128 for the proposed system as well as the SIC without jamming. Note that we cannot plot the SIC and SISO systems with jamming as both fail to achieve a BER of $\leq 10^{-4}$, while the proposed system achieves satisfactory performance. The BER performance loss of the proposed system compared to the SIC system without jamming varies from approximately 3 to $11 \mathrm{~dB}$. The figure also summarises the SNR advantage of massive MIMO, as the higher the number of antennas, the lower the required SNR. However, the rate of decrease of required SNR reduces with the number of antennas, potentially due to the higher correlation of the signals at the antenna array.

Military forces usually employ ultra-high frequency (UHF) radios for communications purposes, with a typical bandwidth of around $250 \mathrm{kHz}$. Fig. 8 represents the throughput using such UHF radios, calculated for SNR $=10 \mathrm{~dB}$ and SNR $=20 \mathrm{~dB}$ for different numbers of antennas from 4 to 128 . This clearly shows that the proposed system is functional and effective even in the presence of high power jamming. The performance is around 4 to 6 Mbps higher compared to the

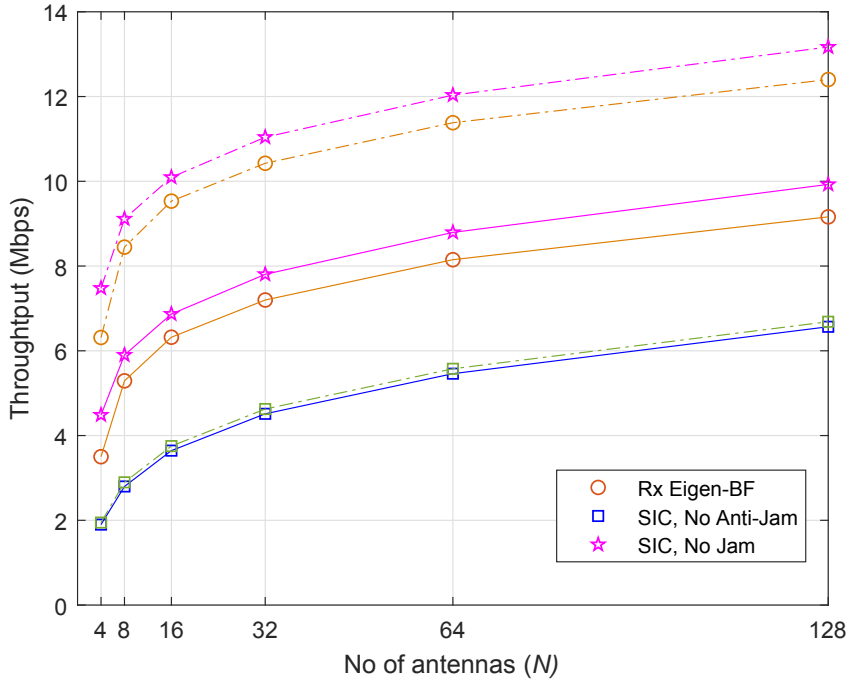

Fig. 8: Throughput for SNR $=10 \mathrm{~dB}$ (solid curves) and SNR $=20 \mathrm{~dB}$ (dash-dotted curves) for different number of antennas $(N)$.

SIC system under jamming, and only around 1 Mbps less than the SIC system without jamming, for both SNR values. The throughput also increases almost linearly with the number of antennas for $N \geq 8$.

Fig. 9 demonstrates how the BER and sum-rate vary with Rician factor. The Rician factors considered here are 1, 4 and 7. It is interesting to see that the sum-rates for both schemes decline with the Rician factor increasing. This is because
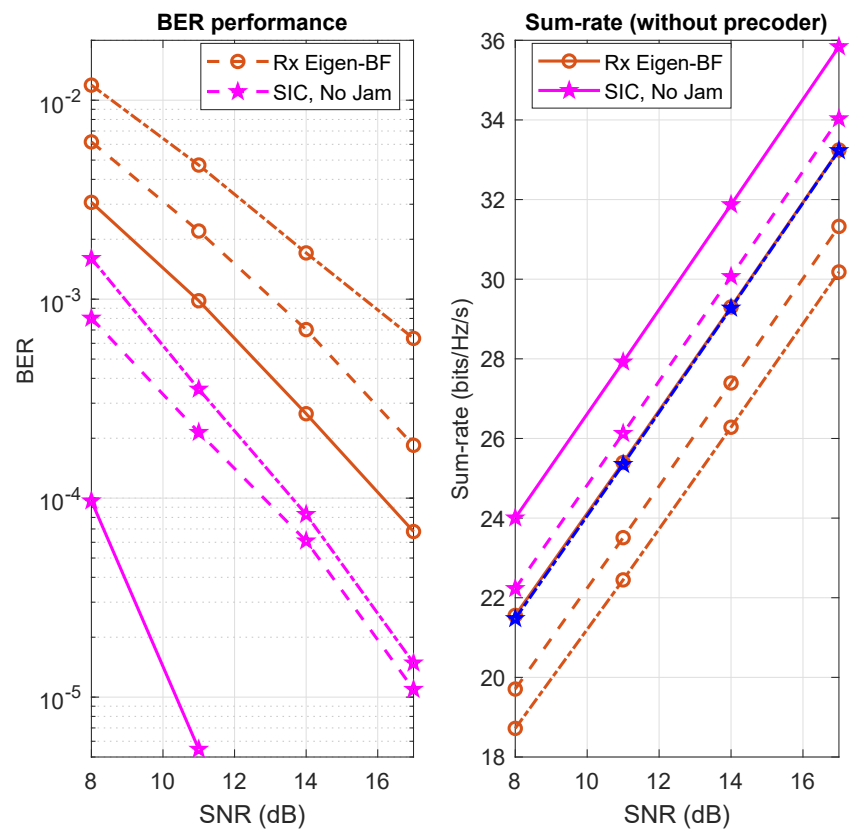

Fig. 9: BER and sum-rate performance for different Rician factors of 1 (solid curves), 4 (dashed curves) and 7 (dashdotted curves). 
when the LoS path becomes less dominating, the channels have more randomness and become less correlated, which leads to higher system capacity. Less correlated jamming channels are also beneficial for jamming suppression in our scheme, particularly when channel estimation error is present, as the singular value will have more uniform absolute values in this case.

\section{CONCLUSions AND Future Work}

We proposed a system for supporting multi-user massive MIMO in tactical ad-hoc networks in a RF contested environment in this paper. We prioritised jamming mitigation over multi-user interference and background noise mitigation to construct a combiner at the receive nodes. Such a combiner spans over the null space of the jamming channels and has the capability to fully remove jamming signals under the assumption that their channels are perfectly known. In addition, we proposed algorithms for determining a linear mapping matrix based on the null space, and designed the zero forcing precoder based on the equivalent channel each receiver node feeds back to the transmit node. We then considered a static tactical network scenario in a contested environment and used extensive simulations to validate the proposed system. The results demonstrated that the proposed jamming mitigation algorithm is capable of effectively mitigating the effects of jamming while supporting multi-user massive MIMO communications. Furthermore, the results also showed that increasing the number of antennas in the array reduces the bit error rate and increases the throughput of the system.

Our next step is to simulate the proposed system in a more dynamic scenario, such as a tactical mobile ad-hoc network (MANET) with mobile jammers. We also intend to investigate more efficient techniques to design the beamforming weights
$\left(\mathbf{W}_{a k}\right)$ at the transmitter and precoding matrix at the receiver.

\section{ACKNOWLEDGMENT}

This research is supported by the Commonwealth of Australia as represented by the Defence Science and Technology Group of the Department of Defence.

\section{REFERENCES}

[1] W. Zhu, B. Daneshrad, J. Bhatia, H. S. Kim, D. Liu, K. Mohammed, R. Prabhu, S. Sasi, and A. Shah, "MIMO Systems for Military Communications," in Military Communications Conference (MILCOM), 2006, pp. 1-7.

[2] B. Daneshrad, "MIMO: The next revolution in wireless data communications," 2008. [Online]. Available: http://defenseelectronicsmag.com/sitefiles/defenseelectronicsmag.com/files/archive/rfdesign.com/militarydefense-electronics/0408DE-MIMO-wireless-revolution.pdf

[3] A. Knopp, R. T. Schwarz, and B. Lankl, "MIMO system implementation with displaced ground antennas for broadband military SATCOM," in 2011 - MILCOM 2011 Military Communications Conference, 2011, pp. 2069-2075.

[4] H. Luo, D. Xu, and J. Bao, "Outage capacity analysis of MIMO system with survival probability," IEEE Communications Letters, vol. 22, no. 6 , pp. 1132-1135, June 2018.

[5] J. R. Pennington and R. K. Martin, "Utilization of inter-block interference in MIMO-OFDM communication systems," in MILCOM 2018 2018 IEEE Military Communications Conference (MILCOM), 2018, pp. $163-168$.

[6] A. Kekirigoda and K.-P. Hui, "Tactical Line-of-Sight MIMO Communication System for Contested Networks," in International Telecommunication Networks and Applications Conference (ITNAC), 2017, pp. 1-6.

[7] E. G. Larsson, O. Edfors, F. Tufvesson, and T. L. Marzetta, "Massive MIMO for next generation wireless systems," IEEE Communications Magazine, vol. 52, no. 2, pp. 186-195, 2014.

[8] E. Björnson, J. Hoydis, and L. Sanguinetti, "Massive MIMO networks: Spectral, energy, and hardware efficiency," Foundations and Trends $₫$ in Signal Processing, vol. 11, no. 3-4, pp. 154-655, 2017. [Online]. Available: http://dx.doi.org/10.1561/2000000093

[9] J. A. Zhang, H. Li, X. Huang, Y. J. Guo, and A. Cantoni, "Userdirected analog beamforming for multiuser millimeter-wave hybrid array systems," in 2017 IEEE 85th Vehicular Technology Conference (VTC Spring), June 2017, pp. 1-5. 